Published version in ULTRAM 161 (2016). DOI 10.1016/j.ultramic.2015.11.015

\title{
Measurement of the permittivity and loss of high-loss materials using a Near-Field Scanning Microwave Microscope
}

\author{
A P Gregory ${ }^{1}$, J F Blackburn ${ }^{1}$, K Lees $^{1}$, R N Clarke ${ }^{1}$, T E Hodgetts ${ }^{2}$, \\ S M Hanham ${ }^{3}$ and N Klein ${ }^{3}$
}

${ }^{1}$ National Physical Laboratory (NPL), ${ }^{2}$ Consultant to NPL, ${ }^{3}$ Imperial College London

\begin{abstract}
In this paper improvements to a Near-Field Scanning Microwave Microscope (NSMM) are presented that allow the loss of high loss dielectric materials to be measured accurately at microwave frequencies. This is demonstrated by measuring polar liquids (loss tangent $\tan \delta \approx 1$ ) for which traceable data is available. The instrument described uses a wire probe that is electromagnetically coupled to a resonant cavity. An optical beam deflection system is incorporated within the instrument to allow contact mode between samples and the probe tip to be obtained. Liquids are contained in a measurement cell with a window of ultrathin glass. The calibration process for the microscope, which is based on image-charge electrostatic models, has been adapted to use the Laplacian 'complex frequency'. Measurements of the loss tangent of polar liquids that are consistent with reference data were obtained following calibration against singlecrystal specimens that have very low loss.
\end{abstract}

Index Terms - Dielectric measurement, microwave microscope, complex frequency.

\section{Introduction}

Two main types of Near-Field Scanning Microwave Microscope (NSMM) [1] are described in the literature: Instruments that are based on the perturbation of the resonance of a cavity that is coupled to a probe tip $[2][3][4][5][6][7][8]$, and instruments that are modified Atomic Force Microscopes (AFMs). AFM-based instruments [9][10][11][12][13] use a Vector Network Analyser (VNA) to measure reflection coefficient data at a reference plane at a tip attached to an AFM cantilever. They are principally used for measuring the conductivity of semiconductors at the nanoscale, but strategies for measurement of permittivity have recently been reported [12][13]. The NSMM which is described in this paper is of the cavity perturbation type. Compared to AFM-based instruments this has a simpler and more calculable geometry as stray fields associated with the cantilever are avoided. It is suitable for measuring the complex permittivity of non-conductive materials, such as ceramics and polymers. The instrument described uses a comparatively large probe (a wire probe with a spherical tip approximately $0.18 \mathrm{~mm}$ in diameter), which enables measurement on the micron scale (which is significantly smaller than the tip diameter as the field is concentrated in the region closest to specimens, especially when the permittivity is high). Q-factor and resonant frequency are obtained by fitting to complex transmission coefficients measured with the VNA, from which complex permittivity may be calculated. A recent review [1] provides more information on the current state of the art of both types of instrument.

In order to make quantitative measurements, the NSMM must be calibrated prior to use against solid reference specimens that have known and reproducible properties, and are isotropic, polished and uniform on a small scale. Single crystal materials with a permittivity of up to 24 that satisfy these requirements are available, however a suitable high loss solid material has not been identified. Carbon-loaded composite materials, for example, can have high loss but do not have the required uniformity at the micron scale. In this paper new approaches to loss measurement are studied. It will be shown that accurate measurements of loss can be obtained following calibration with low-loss single crystals using an implementation of perturbation theory 
under the quasi-static limit that uses the concept of a 'complex frequency' (which mathematically combines resonant frequency and Q-factor). This is tested by making measurements on polar liquids [14], which have high loss and make ideal candidates as reference standards as traceable complex permittivity data is available in the literature [15]. Other workers have used NSMMs to obtain images of aqueous and biological samples in which the probe is immersed [16][17]. In the work described here a well-controlled and calculable geometry was needed, so liquids were contained in a cell with an ultrathin glass window, Fig. 1. The main purpose of this paper is to study algorithms for calibration and extracting $\varepsilon^{\prime}$ and $\tan \delta$, so measurements of uniform specimens at point locations will only be presented. Raster scans can, however, be made in contact mode to allow images to be obtained.

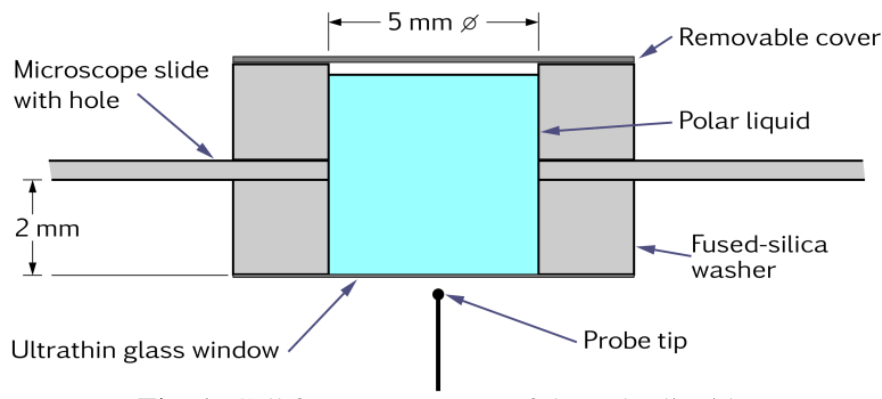

Fig. 1: Cell for measurement of the polar liquids.

The paper is arranged as follows: in Section 2, an overview of the design of the microscope is presented. Section 3 describes a subsystem of the instrument that enables contact mode between tip and specimens to be maintained by shear force detection. Calculation and calibration processes are described in Sections 4 and 5 . A set of measurements on polar liquids are presented in Section 6. An analysis of sensitivity coefficients to inform evaluation of uncertainties is presented in Section 7. Section 8 is the Conclusion.

\section{Overview of the NSMM}

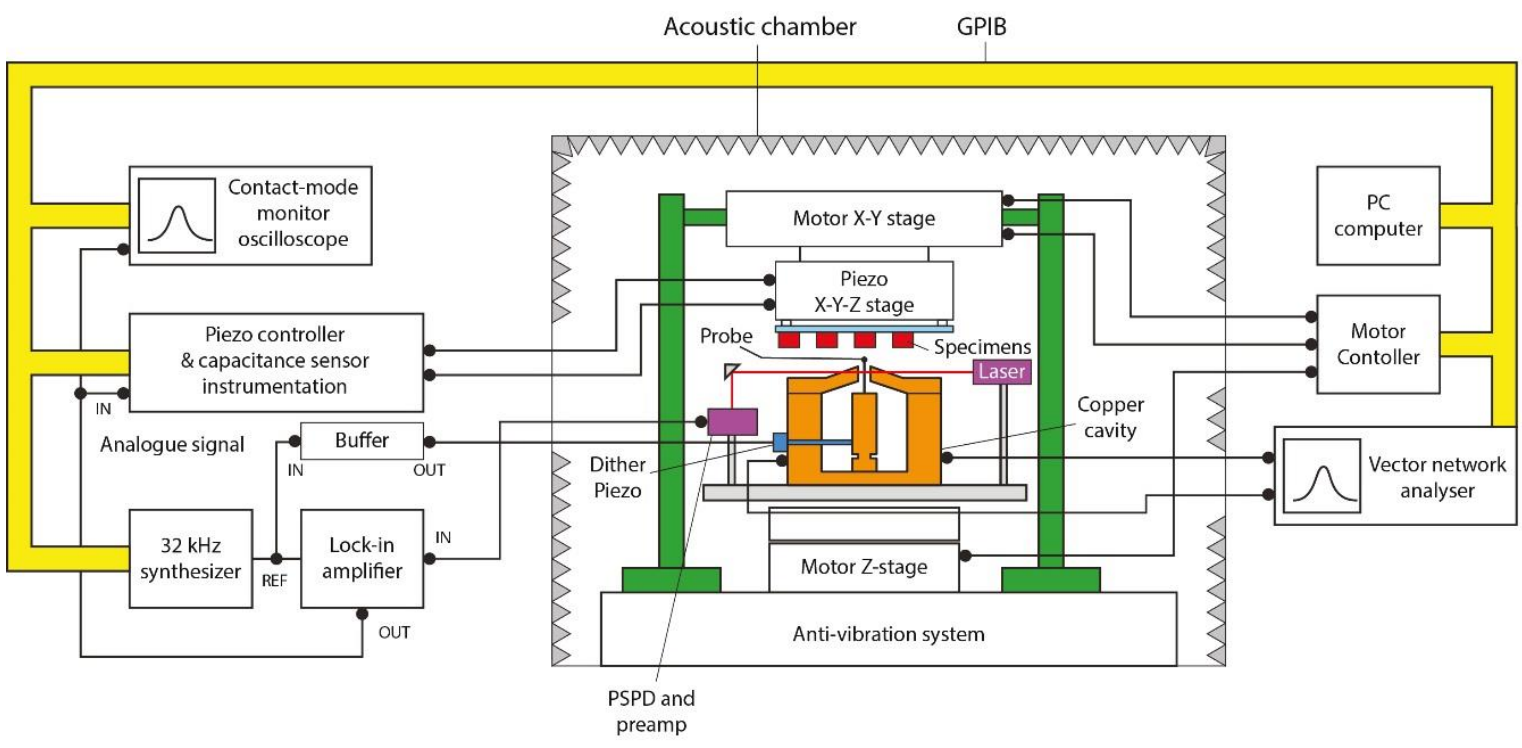

Fig. 2: Schematic of the NSMM 


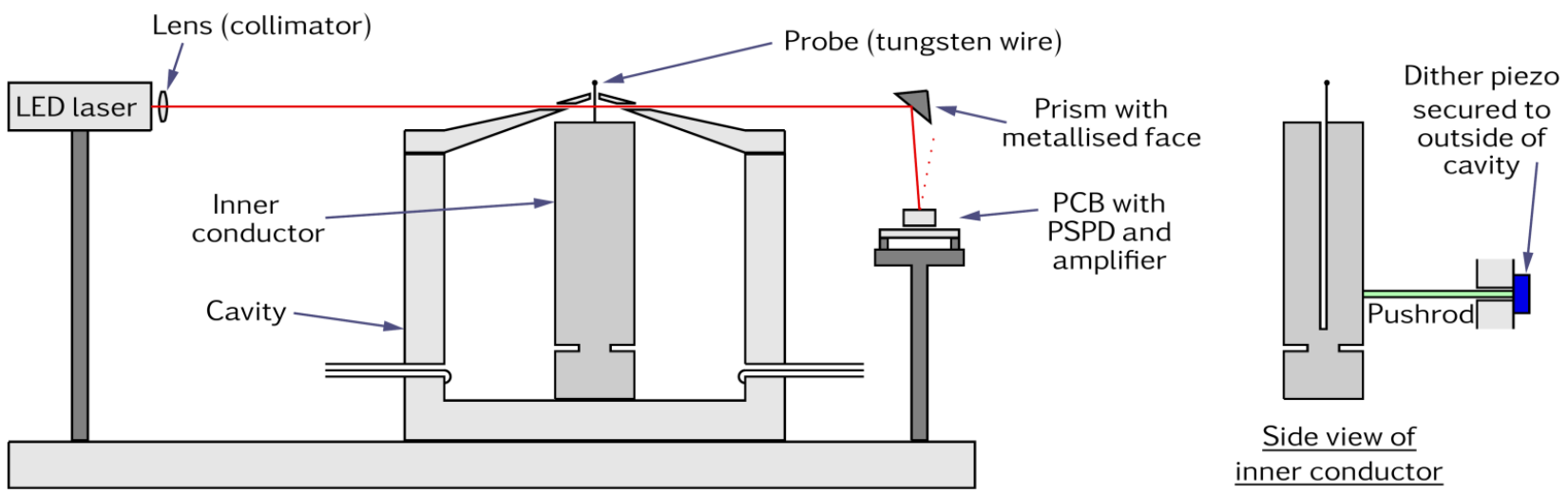

Fig. 3: Optical beam deflection system and quarter-wave microwave cavity. The wire probe is attached to the inner conductor with conducting paint. The top of the cavity is sloped to allow the gap between tip and the specimens placed above it to be observed by using a camera system, which is used to assist setting up.

The NSMM (Fig. 2) is built into an acoustically-shielded chamber with an active anti-vibration table. The combined power supply and control unit for the anti-vibration table is located externally to reduce temperature rises inside the chamber. Shifts in the resonant frequency of the microwave cavity below $100 \mathrm{kHz}$ are significant in these measurements, so mechanical and thermal stability are important. Physik Instrumente (PI) type M-605 $\mathrm{X} \& \mathrm{Y}$ motorized stages with $0.3 \mu \mathrm{m}$ resolution allow large area scans $(50 \times 25 \mathrm{~mm})$. Coarse control of the separation between the specimen and probe tip is provided by means of a motorized $\mathrm{Z}$ stage (range 0 $12.5 \mathrm{~mm})$. Fine-scale movement $(100 \times 100 \times 10 \mu \mathrm{m})$ of the specimens is provided by a three-axis closed-loop piezo stage (PI 733.3CD). A subsystem based on beam deflection (see Section 3) can be used as part of a feedback circuit to control the $\mathrm{Z}$ axis to obtain contact mode. The movement range of the $\mathrm{Z}$ axis of the piezo stage is only $10 \mu \mathrm{m}$, so if contact mode is lost it is unlikely that significant damage will occur. The piezo stages have position sensors based on capacitance measurement which can be read via the GPIB interface.

Specimens (including calibration specimens) are attached to a fused silica substrate (a slide for an optical microscope) using a wax or adhesive. Small magnets are used to attach the slide to the piezo XYZ stage. The control software enables measurement positions to be set up manually and stored. This is achieved by using a joystick attached to the motor controller (PI C-848) to set Z-axis height and a CCD camera with a zoom lens to view the tip and specimen. The motorized XYZ stages can be moved to any of the stored positions using buttons on the software interface. This allows measurements to be made in the minimal amount of time (to reduce drift) and with minimal disturbance to the system. The motorized $\mathrm{Z}$ stage can be raised in micrometer steps via the software user-interface to allow the user to adjust the specimen/tip separation.

The resonator is a coaxial cavity (Fig. 3) with coupling loops that are adjusted to give weak coupling (insertion loss $\approx 36 \mathrm{~dB}$ ). The cavity resonant frequency and Q-factor are obtained by the following procedure: A VNA (Rohde and Schwarz ZVB20) is used to make measurements of the complex transmission coefficient of the cavity over a swept frequency range. These form a circular arc (often referred to as a Q-circle) if plotted on a polar chart. By using a resonance model the resonant frequency $f_{r}$, loaded Q-factor $Q_{L}$, and the Q-circle diameter $d$ can be obtained by a weighted fit [18]. To improve accuracy the leakage vector, which accounts for signal paths between the two ports of the VNA that bypass the resonant circuit, is also fitted. Prior to measurement of Q-factor a preliminary sweep is used to set the VNA frequency span to an optimum range: $f_{r} \pm f_{r} / Q_{L}$. The settings of the VNA used for the measurements presented in this paper were as follows: IFBW $1 \mathrm{kHz}$, averaging factor 1 , source power $0 \mathrm{dBm}, 51$ points, sweep time $0.5 \mathrm{~s}$. For these settings the standard deviation of repeated $Q$-factor measurements is approximately \pm 1 . As the coupling factors at each port were approximately equal, unloaded Q-factor is given by $Q_{L} /(1-d)$ [18]. The VNA is used uncalibrated, but to improve accuracy $d$ is normalised to the magnitude of the transmission coefficient measured when the resonator is replaced by a direct connection.

The cavity is shaped so as to enable an optical beam deflection system to be constructed, though this is achieved at the cost of a reduction in Q-factor and the generation of undesirable higher-order modes. These cause a reduction in the Q-factor and an increase in the effect of higher-modes. At the lowest resonant frequency $(1.21 \mathrm{GHz})$, corresponding to a cavity length of $\lambda / 4$, the cavity resonance was observed to be very well-shaped - 
this is evidenced by the fact that if the if the VNA sweep range is set to encompass the left- or right-hand side of the peak only [18] the same Q-factor is fitted to within $0.1 \%$. Q-factors between 700 and 1500 were obtained, depending on how well-centred the wire probe is in the hole in the top of the cavity (to which it is very sensitive). The next resonance, at $3 \lambda / 4(3.66 \mathrm{GHz})$, was slightly asymmetric; perhaps due to a weak interfering mode. The difference in Q-factor fitted to the left- and right-hand sides of the resonance was $4 \%$. This mode was

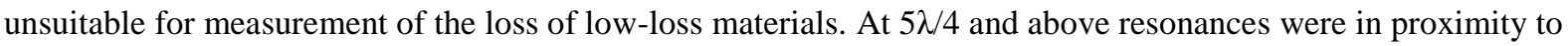
higher-order modes which made them unusable. The higher-order modes couple little energy out of the cavity via the wire probe and so are not useful in this experiment.

The wire probe (tungsten, $0.13 \varnothing \mathrm{mm})$ passes through a small hole $(1 \varnothing \mathrm{mm})$ in the cavity lid. A spherical tip is required to produce a calculable geometry (Section 4). This was fabricated by Electro-Discharge Machining (EDM) [19], which is an appropriate method as it produces a sphere that has a smooth surface (roughness $\sim 100 \mathrm{~nm}$ can be achieved). The tips were nominally $0.18 \mathrm{~mm}$ in diameter. The sphericity of tips is not quite as high as achieved for the smaller-sized tips described in reference [19].

\section{Beam deflection system}

The instrument requires measurements with the probe tip and the surface of the specimen in 'contact mode' in which the gap is $₹ 10 \mathrm{~nm}$. This is obtained by detection of the shear force when the probe tip is subject to smallamplitude vibrations (and is therefore distinct from 'contact mode' in AFM systems in which the tip touches the surface). A highly-sensitive detector is used to obtain a voltage that varies monotonically with the shear-force interaction [20][21][22] between the tip and specimen. This is connected to the piezo-controller (PI E-710), which has built-in closed-loop control circuits that can be used to maintain contact mode. In previously-reported work [23] a tuning fork was used for detecting shear force, however a method based on optical beam deflection [5][20] has been found to be easier to set up. In the present design the cavity inner conductor (a brass rod $10 \varnothing \times$ $50 \mathrm{~mm}$ ) has saw cuts $(0.3 \mathrm{~mm}$ width) to create a tuning fork structure (Fig. 3). This has a higher mechanical Qfactor $(\approx 400)$ than a cantilever-based design [23] and much less dependence on aspects such as how tightly components in the system are bolted down. The wire probe was attached to the brass rod with conducting paint. A ruby jewel bearing can be used to improve the strength of the joint. Small holes in the walls of the cavity $(1.5 \varnothing \mathrm{mm})$ allow illumination of the wire with an amplitude-stabilised red $1 \mathrm{~mW}$ laser (Edmund Optics \#53227). This design enables the protruding length of the wire probe to be kept to a minimum. A lens (built into the laser) allows focus adjustment. Vibrations are excited by means of a piezo transducer (Thorlabs TA0505D024W) on the outside of the cavity and detected using the shadow of the laser beam due to the wire seen in transmission. A push rod with low dielectric loss (fused silica etc.) is used to couple vibrations to the inner conductor. A low-frequency synthesizer (Yokogawa FG200) is used to supply a sine-wave signal at the resonant frequency $(1.3 \mathrm{kHz}$ ). A buffer amplifier (op-amp type OPA452) is used to drive the piezo transducer at $1 \mathrm{~V}$ RMS. The corresponding movement of the piezo transducer is $\approx 40 \mathrm{~nm}$ RMS, which in this experiment does not result in significant modulation of the complex transmission coefficient measured by the VNA. A Position Sensitive Photodiode [PSPD] (First Sensor QP50-6SD2-500741) serves as the detector. By adjustment of the output DC offset of the lock-in amplifier and the synthesizer frequency, a 'lock' to contact mode can be established.

In locked mode the synthesizer is used in $\mathrm{CW}$, but it is also informative to use it in swept mode to allow the Lorentzian response to be viewed using an oscilloscope. Fig. 4 shows the response of the beam deflection as a function of frequency. The quantity $V_{p}$ (defined on the Figure) is chosen to give a stable lock (typically it is set to between $1 / 3$ and $1 / 2$ of $V_{h}$ ). In lock mode the piezo $\mathrm{Z}$ reading shows no significant variation (compared to the stability of readings, $\pm 20 \mathrm{~nm}$ ) as $V_{p}$ is varied between 0.3 and $4 \mathrm{~V}$ (for $V_{h}=7 \mathrm{~V}$ ). Approach curves (Fig. 5) show sharp gradients for small gaps so the accuracy of the contact mode measurement is rather important. 


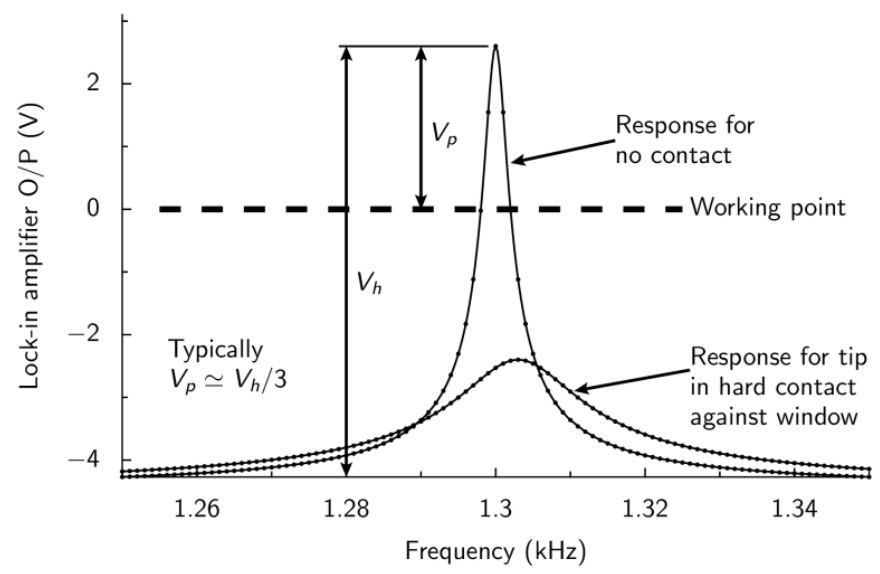

Fig. 4: Frequency response of the beam deflection system measured with the liquid cell filled by ethanediol. For solid reference specimens the peak is further reduced in hard contact. The lock to contact mode is achieved at the working point $(0 \mathrm{~V})$ which can be adjusted using the offset voltage setting of the lock-in amplifier. There are two possible CW lock frequencies. The responses shown are simulated but illustrate the response observed on an oscilloscope with a sweep time of a few seconds (ringing occurs if the sweep is too fast).

\section{Calculation}

Complex permittivity ${ }^{1}\left(\varepsilon^{*}\right)$ can be determined from measurements of the Q-factor and resonant frequency of the cavity respectively unperturbed and with the probe tip in proximity to the specimen. A full analysis would model the entire structure of cavity and the probe tip, but this is very challenging to implement. A simpler method developed by Gao and Xiang (GX) [2] is used in this paper. This enables $\varepsilon^{\prime}$ to be determined using an electrostatic method based on images charges. The method is assumed to remain valid at RF frequencies because the tip dimension is very small compared to the free space RF wavelength. Image charge methods allow field distributions under Maxwellian boundary conditions to be found analytically for conductors and dielectrics that have simple geometrical shapes - see reference [24] for example. GX assume a tip that is a perfect sphere in proximity to a planar dielectric specimen of infinite extent. The perturbation in the cavity resonant frequency $\left(f_{r}\right)$ is derived as:

$$
\frac{\Delta f_{r}}{f_{r}}=-A \sum_{n=1}^{\infty} \frac{b t_{n}}{1+\frac{g}{R_{o}}+a_{n}^{\prime}},
$$

where $A$ is a scaling constant, $g$ is the gap between the probe tip and specimen, $R_{o}$ is the tip radius and $b=$ $\left(\varepsilon^{\prime}-1\right) /\left(\varepsilon^{\prime}+1\right)$. The quantities $t_{n}$ and $a_{n}^{\prime}$ are calculated by simple formulas - see reference [2] for details. Restricting the summation to the first 100 terms yields ample accuracy for the measurements discussed in this paper. Calculation of $\varepsilon^{\prime}$ from measured frequency shifts requires the use of iterative methods, as an explicit expression for $\varepsilon^{\prime}$ cannot be obtained.

\footnotetext{
${ }^{1}$ Expressed by $\varepsilon^{*}=\varepsilon^{\prime}-\mathrm{j} \varepsilon^{\prime \prime}$ where $\varepsilon^{\prime}$ and $\varepsilon^{\prime \prime}$ are both positive. Dielectric loss is characterised by the loss tangent, $\tan \delta=\varepsilon^{\prime \prime} / \varepsilon^{\prime}$.
} 


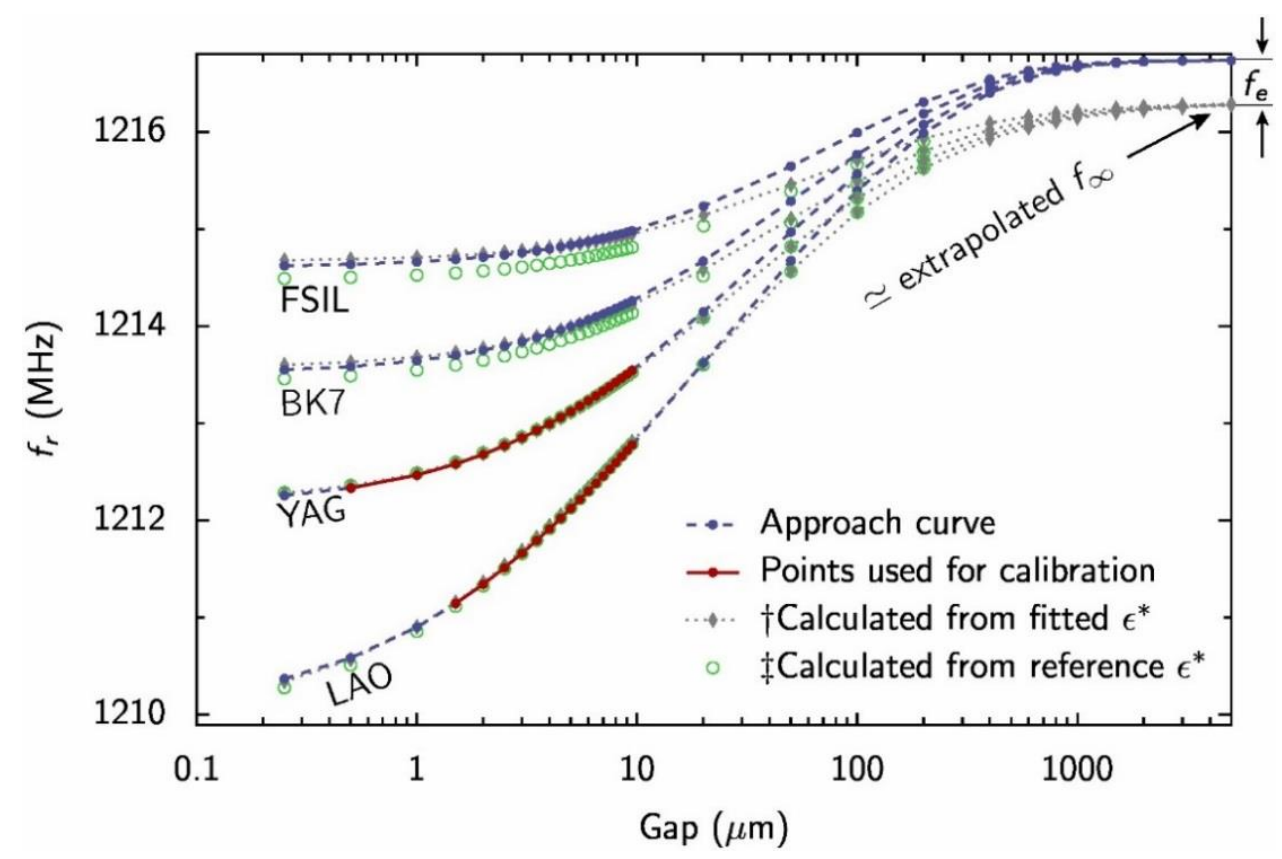

(a) Cavity resonant frequency

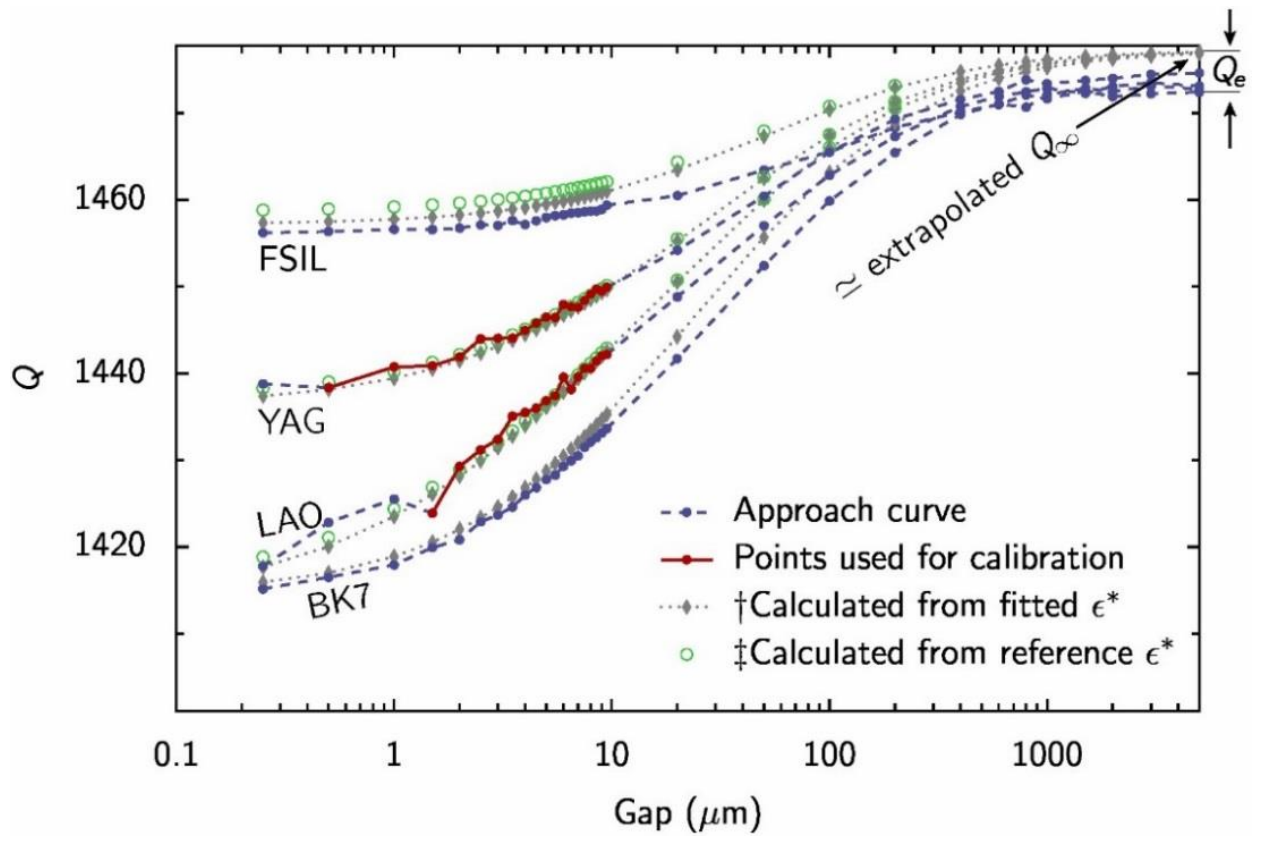

(b) Cavity unloaded Q-factor

Fig. 5: Approach curves measured using the quarter-wave cavity. A subset of the YAG and LAO data are used for calibration (marked in red). The frequency measured when gap $\rightarrow \infty$ is observed to be markedly higher than the value fitted by the calibration $\left(f_{\infty}\right)$. The GXCF calibration algorithm (see Section 5 ) was used to create these graphs. The calibration coefficients are $R o(103 \mu \mathrm{m})$, and $f_{\infty}(1.2163+\mathrm{j} 0.00041 \mathrm{GHz})$ and $A(0.003007-\mathrm{j} 0.000008)$.

† Calculated from $\varepsilon^{*}$ fitted to the approach curve (points in range 0-10 $\mu \mathrm{m}$ only) using the calibration data. Fitted $\varepsilon^{\prime}$ values are : 3.42 (FSIL), 5.95 (BK7), 10.62 (YAG) and 23.29 (LAO).

Fitted $\tan \delta$ values are : 0.0006 (FSIL), 0.0049 (BK7), 0.0001 (YAG) and 0.0003 (LAO).

‡ Calculated from the reference value of $\varepsilon^{*}$ (Table 1 ) using the calibration data. For clarity, points at gaps $>200 \mu \mathrm{m}$ are omitted. Q-factor data for the BK7 specimen is omitted as reference data for tan $\delta$ is only available at $4 \mathrm{GHz}$. 
In principle, $\varepsilon^{\prime}$ can be determined from measurements in contact mode and at a gap that is sufficient to ensure that it does not perturb the resonant frequency of the cavity (a gap of $5 \mathrm{~mm}$ is sufficient). Calibration measurements on one (or more) reference specimens that have known complex permittivity are also needed. However observations of approach ${ }^{2}$ curves show that the frequency measured at a large gap (5 mm) is higher than the value $f_{\infty}$ extrapolated from measurements at small gaps (the discrepancy is marked by $f_{e}$ in Fig. 5). Two possible reasons for this can be suggested: (i) departures from sphericity of actual probe tips and (ii) inaccuracy of the idealised electrostatic model: thus specimen volumes are finite and stray fields are present as the tip is not an isolated sphere. If the cavity is disassembled and put back together, then in subsequent experiments it is found that $f_{e}$ may have changed, even if the same tip is used. It is intended that calibrations should be applicable to image scans in contact mode, so they are carried out using approach curves measured over a small range of gaps (up to $10 \mu \mathrm{m}$ ). This is also the most sensitive range. The (extrapolated) unperturbed frequency is determined by fitting the approach-curve data to the eqn. (1). $R_{o}$ and $A$ are also fitted. The value of $R_{o}$ fitted by the calibration is typically in the range 0.10 to $0.11 \mathrm{~mm}$, which is comparable to the physical radius of the tip.

Gao, Hu et al (GH) [3] published an image-charge method for calculating the frequency shift produced by a two-layer specimen. This was required to account for the window for measurement on liquids contained by the liquid cell (Fig. 1), but can be applied to the measurement of thin films also. The calculation requires traversal of a binary tree in which each node is represented by a data record (e.g. a struct in the language C) that contains various data, including the size of the induced image charge. The size of the induced charges diminishes as the tree expands. The number of nodes is made finite by introducing a lower limit to the induced image charge below which nodes do not beget new nodes. To reduce the computer memory footprint a non-recursive method for traversing the binary tree is used. A combination of a small gap and a thin window (or film) results in a very large binary tree for high permittivity samples. For this work calculations at small gaps are unavoidable, but if the window thickness is over $5 \%$ of the diameter of the spherical tip the tree is a manageable size and accurate results can be obtained in an acceptable time. Numerical experiments demonstrated that the chosen limit of the induced charge was small enough to avoid significant computational error.

GX provide a formula for obtaining dielectric loss from the shifts in Q-factor and resonator caused by a sample,

$$
\Delta\left(\frac{1}{Q}\right)=-(B+\tan \delta) \cdot \frac{\Delta f_{r}}{f_{r}}
$$

in which $B$ is calibration constant which represents Ohmic loss caused by charging current flowing along the wire. In results presented in Section 5, $\tan \delta$ is calculated using eqn. (2) at a range of gaps and then averaged. According to GX, eqn. (2) is applicable only for measurement of the loss of low loss materials. Kimber et al [25] studied the GX method of determining dielectric loss and, using equivalent circuits. They show that, for a tungsten wire probe, the measured dependence of $Q^{-1}$ on $\varepsilon^{\prime}$ is orders of magnitude larger than anticipated due to a loss mechanism that could not be identified. An alternative method of determining dielectric loss that has not, to the authors' knowledge, previously been applied to microwave microscopes is to use the idea that the Qfactor and resonant frequency can be mathematically combined into a Laplacian 'complex frequency',

$$
f_{r}^{*}=f_{r}\left(1+\frac{\mathrm{j}}{2 Q}\right)
$$

where $f_{r}$ and $Q$ are the measured (real) resonant frequency and Q-factor. The complex frequency describes resonant frequency and energy loss in damped harmonic oscillators [26][27]. As a concept it is fairly little known outside purely theoretical contexts and there are not many publications that make use of it. However, its use in the context of resonant electromagnetic modes in cavities is described in detail by Collin [27]. His chief concern is with the case where the losses in the resonant cavity are due to imperfect conductivity of its walls, but his analysis goes through essentially unchanged whenever there is an identifiable surface or volume conduction current with associated Ohmic loss. In every such case we find that lossless complex exponential time behaviour of the form $\exp \left(j 2 \pi f_{r} t\right)$ is replaced by lossy complex exponential time behaviour $\exp \left(j 2 \pi f_{r}^{*} t\right)$, where the real and complex frequencies are connected with each other through equation (3), with a consistent definition of $Q$. (Collin's analysis also establishes a distinction between the real frequency $f_{r}$ and the complex frequency's real

\footnotetext{
${ }^{2}$ Measurements as a function of gap are referred to as approach curves throughout this paper. They are measured as the probe tip is retracted in steps after contact mode has been established.
} 
part $f_{r}{ }^{\prime}$, but this is not specifically considered here because the actual difference between these frequencies is of relative order $Q^{-2}$ rather than $Q^{-1}$ and so is too small to be significant when $Q \approx 1000$ ). In the microwave microscope the complex frequency concept is applied by replacing the frequency $f_{r}$ in eqn. (1) by the complex frequency $f_{r}{ }^{*}$, and replacing the permittivity $\varepsilon^{\prime}$ by the complex permittivity $\varepsilon^{*}$. Eqn. (2) is no longer required. The analysis remains in accordance with Maxwell's equations. The NSMM calibration and measurement procedures in essence are unchanged.

The 'complex frequency' method can be applied to GX and GH models for one and two layer specimens respectively. For convenience, in the remainder of the text the GX and GH models with 'complex frequency' will be referred to as the GXCF and GHCF models. For a one-layer specimen, $\tan \delta$ can be found using the GX model and eqn. (2), or alternatively by using the GXCF model. The calibration constant $B$ can if required be incorporated in the GXCF model by replacing $\tan \delta$ by $B+\tan \delta$, or equivalently, by replacing $\varepsilon^{\prime \prime}$ by $\varepsilon^{\prime} B+\varepsilon^{\prime \prime}$. For two layer specimens the GHCF model is the only one available that enables determination of $\tan \delta$; however Ohmic loss in the wire due to charging current must be a function of the properties of both layers so cannot represented by $B$. For the measurements on high-loss liquids presented in this paper $B$ is excluded from the calibration scheme. This approximation can be made as the dielectric loss of the polar liquid samples has the dominant effect on the Q-factor. The unperturbed resonant frequency, $f_{\infty}{ }^{*}$, is necessarily complex. It is given by

$$
f_{\infty}{ }^{*}=f_{\infty}\left(1+\frac{\mathrm{j}}{2 Q_{\infty}}\right),
$$

where $f_{\infty}$ and $Q_{\infty}$ are the resonant frequency and Q-factor extrapolated to gap $g=\infty$. Tests show that GX and GXCF models give very similar results for low loss materials, but they diverge for high loss materials. In principle, the calibration parameter $A$ can be real or complex. The findings of experiments that compare these options are discussed in Section 5.

\section{Calibration and measurement using the GX and GXCF models}

Calibration constants are fitted to measured approach curves (Fig. 5) for reference specimens that have known $\varepsilon^{\prime}$ and $\tan \delta$. For calibration and measurement, readings at precise gaps up to $10 \mu \mathrm{m}$ between probe tip and specimen are required. These are set using the piezo stage, which lifts the specimens. Position sensors built into the stage enable positions to be set very precisely in closed-loop mode (sensor linearity is $0.03 \%$, repeatability of readings is $\pm 0.5 \mathrm{~nm}$ ). The dominant cause of positioning error using the piezo stage is uncertainty in the Zaxis reading corresponding to contact mode set by the optical beam deflection system (the stability and drift of readings in the time that it takes to measure an approach curve is typically $\pm 20 \mathrm{~nm}$ ). Readings at gaps larger than $10 \mu \mathrm{m}$ can be made using a motorised stage to lower the resonator. The maximum positioning error is $0.3 \mu \mathrm{m}$ for these measurements.

Using the unmodified GX model, Ro (real), and $f_{\infty}$ (real) and $A$ (real) and $\mathrm{B}$ (real) are fitted by the calibration. For the GXCF model Ro (real), and $f_{\infty}$ (complex) and $A$ (real or complex) and optionally B (real) are fitted. Once the calibration has been completed, $\varepsilon^{\prime}$ and $\tan \delta$ can be measured in two ways: (i) by fitting to contact mode measurements of frequency and Q-factor, (ii) by fitting to approach curve measurements of frequency and Qfactor. In both cases $f_{\infty}$ is a constant (unfitted parameter). For approach curve measurements it is possible to disregard the calibration value of $f_{\infty}$ and include it in the fit; however it is found that this strategy tends to give greater errors in the fitted $\varepsilon^{\prime}$ and $\tan \delta$. Contact and approach curve methods can both be applied to the calibration measurements themselves which, for an over-defined calibration, provides a check on the calibration accuracy. Specimens must be polished and uniform on the small scale, and thick enough to ensure that eqn. (1) is valid. The calibration reference specimens are $2-\mathrm{mm}$ thick which is enough to act as a semi-infinite half-space for probe tips $0.1 \mathrm{~mm}$ diameter or smaller (with which the microscope is generally used). The measurements described in this paper use probe tips $0.18 \mathrm{~mm}$ diameter to allow penetration of fields through the window of the liquid cell. For these larger tips, numerical experiments using the GHCF model show that the fused silica substrate which supports the specimens does have a marginal effect (some results demonstrating this will be given in Table 3). The materials from which they are made must be isotropic and have known and reproducible properties. Fused silica and single crystals (Table 1) are ideal as low-loss reference materials. BK7 glass has moderate loss; high enough to be measurable with the microscope. This Section compares the calibration 
schemes, and tests the proposition that the loss of BK7 can be measured using the GXCF model after calibration with low loss materials. The results presented were obtained using the resonance at $1.21 \mathrm{GHz}$.

Table 1: Solid reference specimens

\begin{tabular}{|l|l|l|l|}
\hline \multirow{2}{*}{ Material } & \multirow{2}{*}{ Abbrev. } & \multicolumn{2}{|l|}{ Reference data } \\
\cline { 3 - 4 } & & $\boldsymbol{\varepsilon}^{\prime}$ & $\tan \boldsymbol{\delta}$ \\
\hline$*$ Fused silica & FSIL & $3.80 \pm 0.02$ & $\sim 10^{-4}$ \\
\hline$\dagger \mathrm{Y}_{3} \mathrm{Al}_{5} \mathrm{O}_{12}$ & YAG & $10.59 \pm 0.05$ & $\sim 10^{-5}$ \\
\hline$\dagger \mathrm{LaAlO}_{3}$ & LAO & $23.9 \pm 0.2$ & $\sim 10^{-4}$ \\
\hline $\mathrm{BK} 7 \mathrm{Glass}$ & $\mathrm{BK} 7$ & $\$ 6.36 \pm 0.02$ & $0.0063 \pm 0.0002$ \\
\hline
\end{tabular}

*Data from [28]. Uncertainty in $\varepsilon^{\prime}$ shown is representative of the effect of watercontent variations between samples.

$\dagger$ Undoped single crystal. Measurements on wafers using split-post dielectric resonators [29] performed in the authors' laboratory are shown. Uncertainties are presented at $k=2$.

- LAO is known to vary between batches [30] as rare-earth elements are not easily separated. It is also slightly anisotropic and may show twinning. These variations are unlikely to have much significance for this experiment.

$\$$ A disc $(50 \emptyset \mathrm{mm})$ was cut from the same batch as the NSMM specimen then measured using a split-post dielectric resonator [29] at $4 \mathrm{GHz}$. Uncertainties are presented at $k=2$.

Approach curves are measured under computer control. While the most useful data is for gaps of up to $10 \mu \mathrm{m}$, a measurement of an unperturbed reading with a large gap $(5 \mathrm{~mm})$ is always made to act as a reference to allow drift between measurements to be monitored and, if necessary, corrected for. It is not otherwise used in the calculations for calibration, nor in the measurement of specimens. A gap in contact mode of $\approx 10 \mathrm{~nm}$ results in negligible error (see Section 7) for the large tips used in this work. If materials with $\varepsilon^{\prime} \mp 100$ were measured this would no longer be the case.

The measured dependence of $\mathrm{Q}$ on $\varepsilon^{\prime}$ (Table 2) was obtained by measuring reference materials that have low dielectric loss. LAO has similar loss to FSIL, but the change in Q-factor is greater which shows that there is a dependence on $\varepsilon^{\prime}$. The frequency step for the LAO measurement shown between contact mode and a 5-mm gap was approximately $4 \mathrm{MHz}$. Using eqn. (2) it can be seen that not accounting for the dependence of Q on $\varepsilon^{\prime}$ will typically cause errors in the measurement of $\tan \delta$ that are in the range 0.001 to 0.01 . A correction parameter may be obtained as part of the calibration (e.g. the B parameter introduced in eqn. 2). If this approach is used it may be advantageous to use a high permittivity materials for calibration to resolve the correction parameter more accurately. For this reason LAO was used for calibration for the set of measurements on low loss materials given in this Section. If it were desired to measure $\varepsilon^{\prime}$ of a material having $\varepsilon^{\prime}<10$ then more accurate $\varepsilon^{\prime}$ results would generally be obtained using FSIL and YAG as calibration reference materials.

Table 2: Measured unloaded Q-factors for the lowest cavity resonance $(1.21 \mathrm{GHz})$ for low-loss reference materials as a function of gap. Dielectric loss is not large enough to have significant effect on Q-factor

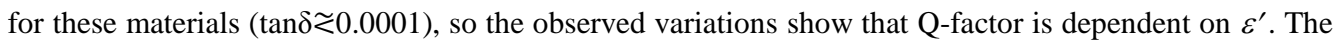
data shown is for a tip that projects $1 \mathrm{~mm}$ beyond the cavity.

\begin{tabular}{|l||l|l|l|}
\cline { 2 - 4 } \multicolumn{1}{c|}{} & \multicolumn{3}{c|}{ Unloaded Q-factor } \\
\hline Gap & FSIL & YAG & LAO \\
\hline$\sim 10 \mathrm{~nm}$ (contact mode) & 1413 & 1397 & 1374 \\
\hline $9 \mu \mathrm{m}$ & 1416 & 1403 & 1397 \\
\hline $5000 \mu \mathrm{m}$ & 1431 & 1430 & 1431 \\
\hline
\end{tabular}

Table 3 shows $\varepsilon^{\prime}$ and $\tan \delta$ results for GX and GXCF models fitted to approach curves measured for FSIL, BK7 and YAG specimens over the range $10 \mathrm{~nm}$ (contact mode) to $9.5 \mu \mathrm{m}$. An approach curve for LAO over 1.5 
to $9.5 \mu \mathrm{m}$ was used for calibration. In general, modifying the ranges of approach curves significantly changes the $\varepsilon^{\prime}$ results, which is an indicator of the fact that the GX model does not perfectly describe the system. Excluding points for $g<1.5 \mu \mathrm{m}$ from the LAO calibration approach curve brings significant improvement to the results shown in Table 3. It was observed that results were very sensitive to whether or not points at $g<1.5 \mu \mathrm{m}$ for the LAO calibration curve were included. This might be associated with imperfections in the shape of the probe tip [19], which would be expected to have the greatest effect for high permittivity specimens. The measurements were made with four different inner-conductor/ wire-probe assemblies, referred to as Tip-1, Tip-2, Tip-3 and Tip-4. For Tip-1 and Tip-2 the wire probe projects 1-mm outside the cavity, for Tip-3 it projects 2-mm, and for Tip-4 it projects 3-mm. Tip-1 and Tip-2 measurements of $\varepsilon^{\prime}$ are consistent for each calibration scheme (although there are systematic differences from the reference data). Only the Tip-1 and Tip-2 measurements are presented, as measurements of $\varepsilon^{\prime}$ with the longer tips were too high by up to $25 \%$. Measurements of $\tan \delta$ showed errors of a similar magnitude. These errors are believed to be due to stray fields associated with a longer tip. It is observed that, for longer probe tips, a Q-factor $>1000$ is more difficult to obtain as it is more sensitive to how well centred the wire is in the hole. Therefore, the Q-factor of measurements with long tips is somewhat variable.

The GXCF model with a complex value for $A$ and with $B$ not fitted and the GX model with $B$ fitted appear to give the most accurate measurements of $\tan \delta$ for BK7 glass. The reference value for BK7 (Table 1) was obtained at $4 \mathrm{GHz}$; it is plausible that at $1.21 \mathrm{GHz}$ it will have changed by $\sim 0.001$. If $\mathrm{A}$ is real, the GXCF model gives an implausibly low value for the fitted $Q_{\infty}$ (c.f. Table 2, which shows data for Tip-1), so negative values of $\tan \delta$ are obtained for fused silica and YAG.

The effect of the finite thickness of the specimens (2-mm) was studied by applying the GHCF model (for $A$ complex and $B$ not fitted) to account for the fused-silica substrate to which the specimens are secured. The GHCF model assumes that the layer farthest from the probe (i.e. the substrate) has infinite thickness; while the actual thickness is only $1 \mathrm{~mm}$, the calculation is nevertheless informative. It is found (see Table 3 ) that the effect of the substrate on $\tan \delta$ is negligible. There is an effect on $\varepsilon^{\prime}$ but it is small, showing that the substrate is not a significant source of error for these measurements. 
Table 3: Measurements of $\varepsilon^{\prime}$ and $\tan \delta$ of solid reference specimens at $1.21 \mathrm{GHz}$ using GX, GXCF and GHCF models. The microscope was calibrated by fitting the coefficients $A, B$ (where used) Ro and $f_{\infty}$ of the chosen model to the measured approach curve for LAO only using points in the gap range 1.5 to $9.5 \mu \mathrm{m}$. Results are obtained by fitting the same model to YAG, FSIL and BK7 approach-curve data for gap ranges $10 \mathrm{~nm}$ (contact mode) to $9.5 \mu \mathrm{m}$. Results shown in brackets are fitted to the contact mode data only. Reference data for these specimens is given in Table 1. Measurements were carried out with two nominally identical tips. A total of four sets of approach curves were recorded as measurements with each tip were made twice. The cavity was dismantled and reassembled between each set of measurements.

\begin{tabular}{|c|c|c|c|c|c|c|c|}
\hline \multirow{4}{*}{\begin{tabular}{|l} 
\\
Tip-1 Meas. 1
\end{tabular}} & \multirow{2}{*}{$Q_{\infty}$} & \multicolumn{2}{|c|}{ Silica } & \multicolumn{2}{|c|}{ YAG } & \multicolumn{2}{|c|}{ BK7 } \\
\hline & & $\varepsilon^{\prime}$ & $\tan \delta$ & $\varepsilon^{\prime}$ & $\tan \delta$ & $\varepsilon^{\prime}$ & $\tan \delta$ \\
\hline & \multicolumn{7}{|c|}{ † GXCF model, A is complex and B is not fitted } \\
\hline & 1431 & $\begin{array}{l}3.80 \\
(3.93)\end{array}$ & $\begin{array}{l}0.0005 \\
(0.0004)\end{array}$ & $\begin{array}{l}11.45 \\
(11.86) \\
\end{array}$ & $\begin{array}{l}0.0000 \\
(-0.0006)\end{array}$ & $\begin{array}{l}6.65 \\
(6.89) \\
\end{array}$ & $\begin{array}{l}0.0047 \\
(0.0047)\end{array}$ \\
\hline Tip-1 Meas. 2 & 1427 & $\begin{array}{l}3.66 \\
(3.78)\end{array}$ & $\begin{array}{l}0.0004 \\
(0.0003)\end{array}$ & $\begin{array}{l}11.34 \\
(11.70)\end{array}$ & $\begin{array}{l}-0.0005 \\
(-0.0002)\end{array}$ & $\begin{array}{l}6.46 \\
(6.72)\end{array}$ & $\begin{array}{l}0.0047 \\
(0.0047)\end{array}$ \\
\hline Tip-2 Meas. 1 & 1417 & 3.99 & 0.0003 & 11.43 & 0.0000 & 6.66 & 0.0047 \\
\hline \multirow[t]{2}{*}{ Tip-2 Meas. 2} & 1482 & 3.97 & 0.0008 & 11.41 & 0.0003 & 6.63 & 0.0047 \\
\hline & \multicolumn{7}{|c|}{$\dagger$ GXCF model, $A$ is real and $B$ is not fitted } \\
\hline Tip-1 Meas. 1 & 1393 & 3.80 & -0.0047 & 11.45 & -0.0013 & 6.64 & 0.0016 \\
\hline \multirow[t]{2}{*}{ Tip-2 Meas. 1} & 1365 & 3.99 & -0.0055 & 11.43 & -0.0015 & 6.66 & 0.0012 \\
\hline & \multicolumn{7}{|c|}{$\dagger$ GXCF model, $A$ is real and $B$ is fitted } \\
\hline Tip-1 Meas. 1 & 1410 & 3.80 & -0.0062 & 11.45 & -0.0032 & 6.65 & -0.0021 \\
\hline \multirow[t]{2}{*}{ Tip-2 Meas. 1} & 1387 & 3.99 & -0.0074 & 11.43 & -0.0038 & 6.66 & -0.0011 \\
\hline & \multicolumn{7}{|c|}{$\$$ GX model, $A$ is real and $B$ is fitted } \\
\hline Tip-1 Meas. 1 & 1431 & $\begin{array}{l}3.80 \\
(3.93)\end{array}$ & $\begin{array}{l}0.0005 \\
(0.0004)\end{array}$ & $\begin{array}{l}11.45 \\
(11.86)\end{array}$ & $\begin{array}{l}0.0000 \\
(-0.0007)\end{array}$ & $\begin{array}{l}6.64 \\
(6.89) \\
\end{array}$ & $\begin{array}{l}0.0061 \\
(0.0068)\end{array}$ \\
\hline Tip-1 Meas. 2 & 1426 & $\begin{array}{l}3.66 \\
(3.80) \\
\end{array}$ & $\begin{array}{l}0.0006 \\
(0.0005) \\
\end{array}$ & $\begin{array}{l}11.34 \\
(11.70)\end{array}$ & $\begin{array}{l}0.0005 \\
(-0.0002) \\
\end{array}$ & $\begin{array}{l}6.46 \\
(6.72) \\
\end{array}$ & $\begin{array}{l}0.0062 \\
(0.0070)\end{array}$ \\
\hline Tip-2 Meas. 1 & 1412 & 3.99 & -0.0003 & 11.43 & -0.0001 & 6.66 & 0.0058 \\
\hline \multirow[t]{2}{*}{ Tip-2 Meas. 2} & 1473 & 3.97 & 0.0001 & 11.41 & 0.0001 & 6.63 & 0.0056 \\
\hline & \multicolumn{7}{|c|}{$\begin{array}{l}\dagger \text { GHCF two-layer model that includes the fused-silica substrate. } \\
\text { A is complex and B is not fitted }\end{array}$} \\
\hline Tip-1 Meas. 1 & 1431 & 3.65 & 0.0005 & 11.36 & 0.0000 & 6.51 & 0.0048 \\
\hline
\end{tabular}

$\dagger Q_{\infty}$ is derived from $f_{\infty}$ (see eqn. (4)) fitted to the LAO calibration measurement.

$\$$ The GX model does not give $Q_{\infty}$, so the value of $Q_{\infty}$ measured at gap $=5 \mathrm{~mm}$ is used for calculating $\tan \delta$. 


\section{Measurements on polar liquids using the GHCF model}

Table 4: Dielectric data and temperature coefficients (TC) for liquids [15] at $20.5^{\circ} \mathrm{C}$.

\begin{tabular}{|c|c|c|c|c|c|c|c|}
\hline \multirow{2}{*}{ Liquid } & \multirow{2}{*}{$\begin{array}{l}\text { Freq. } \\
\text { (GHz) }\end{array}$} & \multicolumn{3}{|c|}{$\varepsilon^{\prime}$} & \multicolumn{3}{|c|}{$\operatorname{Tan} \delta$} \\
\hline & & Value & Unc. $(k=2)$ & TC (per $\left.{ }^{\circ} \mathrm{C}\right)$ & Value & Unc. $(k=2)$ & $\mathrm{TC}\left(\operatorname{per}^{\circ} \mathrm{C}\right)$ \\
\hline \multirow{2}{*}{ Butan-1-ol } & 1.21 & 4.14 & \multirow{2}{*}{0.04} & \multirow{2}{*}{0.05} & 0.761 & \multirow{2}{*}{0.008} & \multirow{2}{*}{0.012} \\
\hline & 1.22 & 4.13 & & & 0.758 & & \\
\hline \multirow{2}{*}{ Ethanediol } & 1.21 & 22.81 & \multirow{2}{*}{0.12} & \multirow{2}{*}{0.57} & 0.754 & \multirow{2}{*}{0.005} & \multirow{2}{*}{-0.021} \\
\hline & 1.22 & 22.67 & & & 0.758 & & \\
\hline
\end{tabular}

Polar liquids have a loss peak in the microwave region of the spectrum [14]. To obtain maximum loss for NSMM calibration, a polar liquid that has its first-order relaxation frequency close to that of the cavity resonance can be chosen, e.g. butan-1-ol (relaxation frequency $257 \mathrm{MHz}$ at $20^{\circ} \mathrm{C}$ ), ethanol $(829 \mathrm{MHz}$ ), ethanediol $(962 \mathrm{MHz})$ dimethyl sulfoxide $(7.6 \mathrm{GHz})$ or water $(17 \mathrm{GHz})$. The loss tangent reaches its maximum at a frequency slightly higher than the relaxation frequency. Dielectric relaxation occurs over a broad frequency range, so it is not necessary that the loss peak should coincide exactly with the cavity resonant frequency. Traceable reference data is available for a number of organic liquids [15]. The window material used was ultrathin glass ${ }^{3}$. This has a high quality surface finish ${ }^{4}$, and is thinner and stiffer than the PEEK window used in previously reported work [23] (which had a tendency to creep during measurements). Using a Linear Variable Differential Transformer (LVDT) system and gauge blocks, the thickness was measured as $0.031 \mathrm{~mm}$ with a population standard deviation of $0.001 \mathrm{~mm}$ (for pieces from the same batch). The dielectric properties of ultrathin glass were measured at $4.0 \mathrm{GHz}$ with a Split-Post Dielectric Resonator [29]. The average permittivity was $\varepsilon^{\prime} W=5.15 \pm 0.14, \tan \delta_{W}=0.006 \pm 0.001$ (uncertainties at coverage factor $k=2$ ). In this text the subscript $W$ is used for the properties of the window. Measurements on dimethyl sulphoxide (DMSO) contained in a cell with PEEK window were previously reported [23], but this liquid is found to be unsuitable for measurement with an ultrathin glass window.

For measurements on liquids (Tables 5 and 6), the system was calibrated from approach curve data for YAG, FSIL and LAO reference specimens in different combinations. For calibration the GXCF model can be applied; however the GHCF model was used instead because the fused-silica substrate which supports the reference specimens has a small effect, which can then be accounted for. The calibration constant A was taken to be complex and B was not fitted. For calibration the following ranges were used (as these typically give best accuracy): FSIL $10 \mathrm{~nm}$ (contact mode) to $9.5 \mu \mathrm{m}$, YAG $0.5 \mu \mathrm{m}$ to $9.5 \mu \mathrm{m}$, and LAO $1.5 \mu \mathrm{m}$ to $9.5 \mu \mathrm{m}$. The GHCF model was used to obtain $\varepsilon^{\prime}$ and $\tan \delta$ of the polar liquids from measurements in contact mode and also from approach curves, range $10 \mathrm{~nm}$ to $9.5 \mu \mathrm{m}$. Tables 5 and 6 also show a few results computed using liquid data in contact mode only, and from approach curves range $1.5 \mu \mathrm{m}$ to $9.5 \mu \mathrm{m}$.

The polar liquid measurements used the same four tips that were used for measuring solid materials (Section 5). The Tables show that the choice of calibration reference material(s) has significant effect upon results. Using a longer probe tip is observed increase the variability of results between calibrations made with different reference materials.

\footnotetext{
3 'AF 32 ECO' aluminoborosilicate glass from Schott AG.

${ }^{4}$ According to manufacturer's data the surfaces are fire-polished giving typical roughness values below $<1 \mathrm{~nm}$ RMS. Waviness perpendicular to the draw direction of the down-draw manufacturing process is $<400 \mathrm{~nm}$.
} 
Table 5: Measurements of $\varepsilon^{\prime}$ and $\tan \delta$ of butan-1-ol made in a cell with ultrathin glass window. Calibration measurements to determine $A$ (complex), Ro (real) and $f_{\infty}$ (complex) were made on 2-mm thick specimens of reference materials secured to a fused silica substrate. The GHCF model was used - see text for more discussion. Calibration approach-curve ranges: FSIL contact mode (nominally $10 \mathrm{~nm}$ ) to $9.5 \mu \mathrm{m}$, YAG 0.5 $\mu \mathrm{m}$ to $9.5 \mu \mathrm{m}$ and LAO $1.5 \mu \mathrm{m}$ to $9.5 \mu \mathrm{m}$. Liquid-cell approach curve ranges were $10 \mathrm{~nm}$ to $9.5 \mu \mathrm{m}$ except where bracketed. (..) indicates that only the contact-mode measurement was used. [..] indicates that the range was $1.5 \mu \mathrm{m}$ to $9.5 \mu \mathrm{m}$. In each run all measurements (including calibration measurements) were repeated. The three runs for each tip were made without dismantling the cavity.

\begin{tabular}{|c|c|c|c|c|c|c|c|c|}
\hline \multirow[t]{2}{*}{$\begin{array}{l}\text { Calibration } \\
\text { reference } \\
\text { specimens }\end{array}$} & \multicolumn{2}{|c|}{$\begin{array}{l}\text { Butan-1-ol } \\
\text { reference } \\
\text { data at } \\
1.21 \mathrm{GHz}\end{array}$} & \multicolumn{3}{|c|}{ Tip-1 } & \multicolumn{3}{|c|}{ Tip-2 } \\
\hline & $\varepsilon^{\prime}$ & $\tan \delta$ & Run & $\varepsilon^{\prime}$ & $\tan \delta$ & Run & $\varepsilon^{\prime}$ & $\tan \delta$ \\
\hline YAG & \multirow{4}{*}{4.14} & \multirow{4}{*}{0.761} & $\begin{array}{l}1 \\
(1) \\
2 \\
3\end{array}$ & $\begin{array}{l}3.76 \\
(3.97) \\
3.69 \\
3.84\end{array}$ & $\begin{array}{l}0.948 \\
(0.918) \\
0.906 \\
0.902\end{array}$ & $\begin{array}{l}4 \\
(4) \\
5 \\
6 \\
\end{array}$ & $\begin{array}{l}4.02 \\
(4.08) \\
4.19 \\
3.97 \\
\end{array}$ & $\begin{array}{l}0.801 \\
(0.773) \\
0.846 \\
0.851\end{array}$ \\
\hline LAO & & & $\begin{array}{l}1 \\
(1) \\
2 \\
3 \\
\end{array}$ & $\begin{array}{l}4.69 \\
(4.93) \\
4.61 \\
4.25 \\
\end{array}$ & $\begin{array}{l}0.0 .795 \\
(0.792) \\
0.769 \\
0.833 \\
\end{array}$ & $\begin{array}{l}4 \\
(4) \\
5 \\
6 \\
\end{array}$ & $\begin{array}{l}4.60 \\
(4.69) \\
4.86 \\
3.89 \\
\end{array}$ & $\begin{array}{l}0.744 \\
(0.728) \\
0.775 \\
0.844 \\
\end{array}$ \\
\hline $\begin{array}{l}\text { YAG and } \\
\text { LAO }\end{array}$ & & & $\begin{array}{l}1 \\
(1) \\
2 \\
3 \\
\end{array}$ & $\begin{array}{l}3.87 \\
(4.10) \\
3.69 \\
3.80 \\
\end{array}$ & $\begin{array}{l}0.912 \\
(0.894) \\
0.897 \\
0.902\end{array}$ & $\begin{array}{l}4 \\
(4) \\
5 \\
6 \\
\end{array}$ & $\begin{array}{l}3.80 \\
(3.90) \\
4.07 \\
4.09\end{array}$ & $\begin{array}{l}0.849 \\
(0.820) \\
0.866 \\
0.816\end{array}$ \\
\hline $\begin{array}{l}\text { FSIL and } \\
\text { YAG }\end{array}$ & & & $\begin{array}{l}1 \\
(1) \\
{[1]} \\
2 \\
3 \\
\end{array}$ & $\begin{array}{l}4.80 \\
(4.91) \\
{[4.77]} \\
4.63 \\
4.71 \\
\end{array}$ & $\begin{array}{l}0.729 \\
(0.716) \\
{[0.732]} \\
0.713 \\
0.724 \\
\end{array}$ & $\begin{array}{l}4 \\
(4) \\
{[4]} \\
5 \\
6 \\
\end{array}$ & $\begin{array}{l}.44 \\
(4.45) \\
{[4.45]} \\
4.59 \\
4.62 \\
\end{array}$ & $\begin{array}{l}0.720 \\
(0.699) \\
{[0.699]} \\
0.773 \\
0.737 \\
\end{array}$ \\
\hline
\end{tabular}


Table 6: Measurements of $\varepsilon^{\prime}$ and $\tan \delta$ of ethanediol made in a cell with ultrathin glass window. They were fitted to measured approach curves using the GHCF model. Calibration measurements to determine $A$ (complex), Ro (real) and $f_{\infty}$ (complex) were made on 2 -mm thick specimens of reference materials secured to a fused silica substrate. The GHCF model was used - see text for more discussion. Calibration approach-curve ranges: FSIL contact mode (nominally $10 \mathrm{~nm}$ ) to $9.5 \mu \mathrm{m}$, YAG $0.5 \mu \mathrm{m}$ to $9.5 \mu \mathrm{m}$ and LAO $1.5 \mu \mathrm{m}$ to $9.5 \mu \mathrm{m}$. Liquid-cell approach curve ranges were $10 \mathrm{~nm}$ to $9.5 \mu \mathrm{m}$ except where bracketed. (..) indicates that only the contact-mode measurement was used. [..] indicates that the range was $1.5 \mu \mathrm{m}$ to $9.5 \mu \mathrm{m}$. In each run all measurements (including calibration measurements) were repeated. The three runs for each tip were made without dismantling the cavity.

\begin{tabular}{|c|c|c|c|c|c|c|c|c|}
\hline \multirow[t]{2}{*}{$\begin{array}{l}\text { Calibration } \\
\text { reference } \\
\text { specimens }\end{array}$} & \multicolumn{2}{|c|}{$\begin{array}{l}\text { Ethanediol } \\
\text { reference } \\
\text { data at } \\
1.21 \mathrm{GHz}\end{array}$} & \multicolumn{3}{|c|}{ Tip-1 } & \multicolumn{3}{|c|}{ Tip-2 } \\
\hline & $\varepsilon^{\prime}$ & $\tan \delta$ & Run & $\varepsilon^{\prime}$ & $\tan \delta$ & Run & $\varepsilon^{\prime}$ & $\tan \delta$ \\
\hline YAG & \multirow{4}{*}{22.81} & \multirow{4}{*}{0.754} & $\begin{array}{l}10 \\
(10) \\
11 \\
12\end{array}$ & $\begin{array}{l}23.50 \\
(24.26) \\
24.30 \\
24.22\end{array}$ & $\begin{array}{l}0.984 \\
(0.848) \\
0.844 \\
0.875\end{array}$ & $\begin{array}{l}13 \\
(13) \\
14 \\
15\end{array}$ & $\begin{array}{l}23.76 \\
(23.92) \\
24.06 \\
24.15\end{array}$ & $\begin{array}{l}0.0 .853 \\
(0.794) \\
0.731 \\
0.756\end{array}$ \\
\hline $\mathrm{LAO}$ & & & $\begin{array}{l}10 \\
(10) \\
11 \\
12 \\
\end{array}$ & $\begin{array}{l}23.95 \\
(24.75) \\
25.00 \\
24.74 \\
\end{array}$ & $\begin{array}{l}0.639 \\
(0.622) \\
0.600 \\
0.641\end{array}$ & $\begin{array}{l}13 \\
(13) \\
14 \\
15\end{array}$ & $\begin{array}{l}23.03 \\
(24.30) \\
25.01 \\
25.46 \\
\end{array}$ & $\begin{array}{l}0.667 \\
(0.661) \\
0.656 \\
0.627\end{array}$ \\
\hline $\begin{array}{l}\text { YAG and } \\
\text { LAO }\end{array}$ & & & $\begin{array}{l}10 \\
(10) \\
{[10]} \\
11 \\
12\end{array}$ & $\begin{array}{l}23.53 \\
(24.10) \\
{[23.33]} \\
24.05 \\
24.05\end{array}$ & $\begin{array}{l}0.743 \\
(0.694) \\
{[0.765]} \\
0.786 \\
0.798\end{array}$ & $\begin{array}{l}13 \\
(13) \\
{[13]} \\
14 \\
15\end{array}$ & $\begin{array}{l}23.60 \\
(23.86) \\
{[23.45]} \\
24.00 \\
24.04\end{array}$ & $\begin{array}{l}0.731 \\
(0.705) \\
{[0.739]} \\
0.804 \\
0.830\end{array}$ \\
\hline $\begin{array}{l}\text { FSIL and } \\
\text { YAG }\end{array}$ & & & $\begin{array}{l}10 \\
(10) \\
11 \\
12 \\
\end{array}$ & $\begin{array}{l}21.77 \\
(21.59) \\
22.90 \\
22.68 \\
\end{array}$ & $\begin{array}{l}0.468 \\
(0.449) \\
0.522 \\
0.508 \\
\end{array}$ & $\begin{array}{l}13 \\
(13) \\
14 \\
15 \\
\end{array}$ & $\begin{array}{l}23.01 \\
(22.87) \\
23.30 \\
23.00\end{array}$ & $\begin{array}{l}0.589 \\
(0.575) \\
0.578 \\
0.598 \\
\end{array}$ \\
\hline
\end{tabular}




\section{Uncertainties of the polar liquid measurements}

Sections 5 and 6 show that there are significant systematic uncertainties for which there is no comprehensive uncertainty model at present, so standard procedures for evaluating uncertainty based on sensitivity coefficients [31] cannot be applied. A limited evaluation of uncertainty can be made from measurements on reference materials such as those given in this paper. Nevertheless it is still informative to consider causes of uncertainties that we can evaluate by calculation of sensitivity coefficients. It is particularly important to understand how the window parameters (complex permittivity and thickness) affect the measurement on liquids.

Permittivity of polar liquid samples: Polar liquids have high temperature coefficients so the measurement temperature must be known. There was no practical means of measuring the temperature of the liquids in situ, so they were taken to be the same as the environment (between 20 and $21^{\circ} \mathrm{C}$ ). The temperature coefficient data in Table 4 shows that the effect of temperature variations on $\varepsilon^{\prime}$ and $\tan \delta$ is not significant for these measurements. Neither are the uncertainties of the reference data significant. The resonant frequencies were in the range 1.21 to $1.22 \mathrm{GHz}$ for both tips, which does not cause a significant variation in permittivity.

\section{Contact mode gap:}

If $\varepsilon^{\prime}$ is fitted to the contact mode point only, then accounting for the $10 \mathrm{~nm}$ residual gap increases the $\varepsilon^{\prime}$ result by $\approx 1 \%$ for a specimen with $\varepsilon^{\prime} \approx 25$ for the probe tips used in this paper. If permittivity is fitted to approach curve data then the effect of the gap in contact mode is substantially reduced.

\section{Thickness, permittivity and loss of the window:}

Table 7: Estimated Sensitivity coefficients for window data for butan-1-ol. Calculated using data for Run 1 with calibration using YAG and LAO. Subscripts $L$ and $W$ refer to the butan-1-ol and window respectively.

\begin{tabular}{|l|l|}
\hline$\Delta \varepsilon^{\prime}{ }_{L} / \Delta t_{W}=0.03$ per $\mu \mathrm{m}$ & $\Delta \tan \delta_{L} / \Delta t_{W}=-0.01$ per $\mu \mathrm{m}$ \\
\hline$\Delta \varepsilon^{\prime}{ }_{L} / \Delta \varepsilon^{\prime}{ }_{W}=-0.20$ & $\Delta \tan \delta_{L} / \Delta \varepsilon^{\prime} W=-0.02$ \\
\hline$\Delta \varepsilon^{\prime}{ }_{L} / \Delta \tan \delta_{W}=2.0$ & $\Delta \tan \delta_{L} / \Delta \tan \delta_{W}=-0.6$ \\
\hline
\end{tabular}

Table 8: Estimated Sensitivity coefficients for window data for ethanediol. Calculated using data for Run 10 with calibration using YAG and LAO. Subscripts $L$ and $W$ refer to the ethanediol and window respectively.

\begin{tabular}{|l|l|}
\hline$\Delta \varepsilon_{L}^{\prime} / \Delta t_{W}=0.11$ per $\mu \mathrm{m}$ & $\Delta \tan \delta_{L} / \Delta t_{W}=0.04$ per $\mu \mathrm{m}$ \\
\hline$\Delta \varepsilon_{L}^{\prime} / \Delta \varepsilon^{\prime}{ }_{W}=-1.5$ & $\Delta \tan \delta_{L} / \Delta \varepsilon^{\prime}{ }_{W}=-0.3$ \\
\hline$\Delta \varepsilon^{\prime}{ }_{L} / \Delta \tan \delta_{W}=-54$ & $\Delta \tan \delta_{L} / \Delta \tan \delta_{W}=-2$ \\
\hline
\end{tabular}

Tables 7 and 8 show estimated sensitivity coefficients of the measured permittivity and loss tangent of the liquid as a function of the permittivity $\left(\varepsilon^{\prime} W\right)$, loss tangent $\left(\Delta \tan \delta_{W}\right)$ and thickness $\left(\Delta t_{W}\right)$ of the glass window. Uncertainty in the SPDR measurement of $\varepsilon^{\prime} W$ is almost entirely associated with error in $t_{W}$ (so is considered to be a dependent contribution). It is found that $\Delta \varepsilon^{\prime} w / \Delta t w=-0.14$. The dielectric properties of the ultrathin glass window $\left(\varepsilon^{\prime} W=5.15 \pm 0.14, \tan \delta_{W}=0.006 \pm 0.001\right)$ were measured at $4 \mathrm{GHz}$. At the frequency of NSMM measurements, 1.21 to $1.22 \mathrm{GHz}$, the real part of permittivity will not be significantly greater. A small change in the loss would be expected, which is accounted for by increasing $U_{\tan \delta_{W}}$ to 0.002 . This increase is too small to significantly affect the combined uncertainties. 
Uncertainty contributions associated with the window can be combined [31]. The uncertainty associated with the measured permittivity of the liquid the, $U_{\varepsilon_{L}}$, can be evaluated using:

$$
U_{\varepsilon_{L}}=\sqrt{\left(\left(\frac{\Delta \varepsilon_{L}^{\prime}}{\Delta t_{W}}+\frac{\Delta \varepsilon_{L}^{\prime}}{\Delta \varepsilon_{W}^{\prime}} \frac{\Delta \varepsilon_{W}^{\prime}}{\Delta t_{W}}\right) U_{t_{W}}\right)^{2}+\left(\frac{\Delta \varepsilon_{L}^{\prime}}{\Delta \tan \delta_{W}} U_{\tan \delta_{W}}\right)^{2}}
$$

A similar expression is used for evaluating $U_{\tan \delta_{W}}$. Evaluated uncertainties for the liquid measurmeents are given in Table 9 (calculated assuming $U_{t_{W}}=1 \mu \mathrm{m}$ ). These contributions may be compared to the discrepancies between measurements that are obtained when different calibration reference materials are used (Tables 5 and 6). $U_{\tan \delta_{L}}$ for ethanediol is seen to be comparable to the observed discrepancies of the loss. $U_{\tan \delta_{L}}$ for butan-1-ol and $U_{\varepsilon_{L}}$ for both liquids are seen not to be significant.

Table 9: Combined uncertainty contributions associated with the ultrathin glass window (evaluated at $k=2$ ).

\begin{tabular}{|l|c|c|}
\hline & $U_{\varepsilon_{L}}$ & $U_{\tan \delta_{L}}$ \\
\hline Butan-1-ol & 0.004 & 0.01 \\
\hline Ethanediol & 0.3 & 0.1 \\
\hline
\end{tabular}

\section{Conclusion}

A microwave microscope with a wire probe that has a spherical tip has been described. This incorporates an optical-beam deflection system for detection of contact mode. A new approach to loss measurement based using the 'complex frequency' has been developed, which allows the loss of materials that have medium or high loss to be obtained following calibration against reference materials that have very low loss. This provides a solution to the problem that high-loss solid reference materials are not easily obtained. Experimentally, it is has been found that a resolution of measurements of loss angle $\delta$ of the order of a milliradian for bulk materials is obtainable.

To gain understanding of the accuracy of the complex frequency approach the loss of polar liquids was measured in a cell with a window made from ultrathin glass. Systematic errors in measurements of $\varepsilon^{\prime}$ and $\tan \delta$ are observed to depend on the choice of calibration reference materials and the approach curve range. They also depend on the length of the probe tip. Shorter tips tend to give more accurate results, with better repeatability after the cavity has been dismantled and re-assembled. It is observed that the cavity Q-factor is significantly reduced if the wire is off-centre in the $1 \varnothing \mathrm{mm}$ hole in the top of the cavity (especially for the longer tips). There are believed to be several factors which underlie the systematic errors in $\varepsilon^{\prime}$ and $\tan \delta$, including stray field and radiative effects, the finite area and thickness of specimens, and imperfections in the sphericity of probe tips (which make it beneficial to exclude points at small gaps for high-permittivity calibration specimens). Models derived directly from theory that use only a few calibration coefficients have been used here. Modifications to the models to improve accuracy (by adding extra terms) could be tractable, but in this paper satisfactory results are obtained by selection of the ranges of approach curves for the calibration specimens. The accuracy of calibration is often improved if two reference specimens having different permittivity are used.

For this work the comparatively large probe tips $(0.18 \emptyset \mathrm{mm})$ were used to enable penetration of fields through the ultrathin-glass window. Appropriate choice of calibration reference materials enables the best measurement accuracy to be attained; typically, $\pm 10 \%$ for $\varepsilon^{\prime}$ and $\pm 20 \%$ for $\tan \delta$. These estimated accuracies are expected to be valid for the ranges $3<\varepsilon^{\prime}<25$ and $0.005<\tan \delta<1$ at least. The validity of the 'complex frequency' approach has been demonstrated; nevertheless some aspects remain unexplained, 
such as the dependence of $Q$ on $\varepsilon^{\prime}$ [25] for low loss specimens ( $\tan \delta \widetilde{<0.0001) . ~ F u r t h e r ~ t h e o r e t i c a l ~ s t u d y ~}$ of these results is merited.

\section{Acknowledgement}

The NSMM was developed under the project "Electromagnetic Materials Measurements for Industrial Applications (EMINDA)". This was a 3-year project under the European Metrology Research Programme (EMRP) under Grant Agreement No. 217257. The EMRP is jointly funded by the EMRP participating countries within EURAMET and the European Union. Additional funding from the IRD programme of the National Measurement Office of the UK Government Department of Business, Innovation and Skills supported the development of the beam deflection systems.

The authors acknowledge assistance from a number of NPL colleagues; in particular M. Stewart and A. Yacoot for useful discussions on the beam deflection system, and M. A. Barnett and G. J. Hill who tested ultrathin glass for absorption of liquids by using an admittance cell. D.-Y. Sheu of the National Taipei University of Technology, Taiwan, supplied the EDM probe tips.

\section{References}

[1] A. Imtiaz, T. M. Wallis and P. Kabos, "Near-field scanning microwave microscopy: An Emerging Research Tool for Nanoscale Metrology," IEEE Microw. Mag., vol. 15, no. 1, pp. 52-64, 2014, http://dx.doi.org/10.1109/MMM.2013.2288711

[2] C. Gao and X.-D. Xiang, "Quantitative microwave near-field microscopy of dielectric properties," Rev. Sci. Instrum., vol 69, no. 11, pp. 3846-3851, 1998, http://dx.doi.org/10.1063/1.1149189

[3] C. Gao, B. Hu, P. Zhang, M. Huang, W. Liu and I. Takeuchi, "Quantitative microwave evanescent microscopy of dielectric thin films using a recursive image charge approach," Appl. Phys. Lett., vol. 84, no. 23, pp. 4647-4649, 2004, http://dx.doi.org/10.1063/1.1759389

[4] H.-F. Cheng, Y.-C. Chen and I.-N. Lin, "Evanescent microwave probe study on dielectric properties of materials," J. Eur. Cer. Soc., vol. 26, pp. 1801-1805, 2006, http://dx.doi.org/10.1016/j.jeurceramsoc.2005.09.034

[5] V. V. Talanov, A. Scherz, R. L. Moreland and A. R. Schwartz, "A near-field scanned microwave probe for spatially localized electrical metrology”, Appl. Phys. Lett., vol 88, pp. 134106, 2006, http://dx.doi.org/10.1063/1.2189147

[6] S. Hanham, A. Gregory, S. Maier and N. Klein, "A dielectric probe for near-field millimeter-wave imaging," 37th International Conference on Infrared, Millimeter, and Terahertz Waves (IRMMW-THz), 2012, http://dx.doi.org/ 10.1109/IRMMW-THz.2012.6380378

[7] D. J. Barker, T. J. Jackson, P. M. Suherman, M. S. Gashinova and M. J. Lancaster, "Uncertainties in the permittivity of thin films extracted from measurements with near field microwave microscopy calibrated by an image charge model", Meas. Sci Tech., vol 25, pp. 105601, 2014, http://dx.doi.org/10.1088/0957-0233/25/10/105601

[8] A. Imtiaz, T. Baldwin, H. T. Nembach, T. M. Wallis, and P. Kabos, "Near-field microwave microscope measurements to characterize bulk material properties", Appl. Phys. Lett, vol. 90, pp. 243105, 2007, http://dx.doi.org/10.1063/1.2748307

[9] A. Imtiaz, T. M. Wallis, S.-H. Lim, H. Tanbakuchi, H.-P. Huber, A. Hornung, P. Hinterdorfer, J. Smoliner, F. Kienberger and P. Kabos, "Frequency-selective contrast on variably doped p-type silicon with a scanning microwave microscope", J. Appl. Phys. vol.111, pp. 093727, 2012, http://dx.doi/org/10.1063/1.4716026

[10] A. Tselev, N. V. Lavrik, I. Vlassiouk, D. P. Briggs, M. Rutgers, R. Proksch and S. V. Kalinin, "Near-field microwave scanning probe imaging of conductivity inhomogeneities in CVD graphene", Nanotechnology vol. 23, pp. 385706, 2012, http://dx.doi.org/10.1088/0957-4484/23/38/385706

[11] A. Karbassi, D. Ruf, A. D. Bettermann, C. A. Paulson, D. W. van der Weide, H. Tanbakuchi, and R. Stancliff, "Quantitative scanning near-field microwave microscopy for thin film dielectric constant measurement", Rev. Sci. Instrum. vol 79, pp. 094706, 2008, http://dx.doi.org/10.1063/1.2953095

[12] G. Gramse, M. Kasper, L. Fumagalli, G. Gomila, P. Hinter-dorfer and F. Kienberger, "Calibrated complex impedance and permittivity measurements with scanning microwave microscopy," Nanotechnology, vol. 25, no. 14, pp. 145703, 2014, http://dx.doi.org/10.1088/0957-4484/25/14/145703

[13] J. Hoffmann, G. Gramse, J. Niegemann, M. Zeier and F. Kienberger, "Measuring low loss dielectric substrates with scanning probe microscopes," Appl. Phys. Lett., vol. 105, pp. 013102, 2014, http://dx.doi.org/10.1063/1.4886965 
[14] A. P. Gregory and R. N. Clarke, "A review of RF and microwave techniques for dielectric measurements on polar Liquids," IEEE Trans. Diel. and Elec. Insulation, vol. 13 no. 4 pp. 727-743 2006, http://dx.doi.org/ 10.1109/TDEI.2006.1667730

[15] A. P. Gregory and R. N. Clarke, "Tables of the complex permittivity of dielectric reference liquids up to $5 \mathrm{GHz}, ”$ NPL Report MAT 23, 2012.

[16] K. Haddadi, S. Gu, T. Lasri, "Sensing of liquid droplets with a scanning near-field microwave microscope", Sensors and Actuators A: Physical, vol. 230, 170-174, 2015, http://dx.doi.org/10.1016/j.sna.2015.04.028

[17] M. Farina, A. Di Donato, D. Mencarelli, G. Venanzoni, and A. Morini, "High Resolution Scanning Microwave Microscopy for Applications in Liquid Environment", IEEE Microwave and Wireless Components Letters, vol. 22, no. 11, 595-597, 2012, http://dx.doi.org/10.1109/LMWC.2012.2225607

[18] A. P. Gregory, "Q-factor measurement using a vector network analyser," NPL Report MAT 58, 2016 (to be published).

[19] D.-Y. Sheu, "Micro-spherical probes machining by EDM," J. Micromech. Microeng., vol. 15, no. 1, pp. 185189, 2005, http://dx.doi.org/ 10.1088/0960-1317/15/1/026

[20] A. Yacoot and L. Koenders, "Aspects of scanning force microscope probes and their effects on dimensional measurement," J. Phys. D: Appl. Phys., vol. 41, 103001, 2008, http://dx.doi.org/10.1088/0022-3727/41/10/103001

[21] L. Beaulieu, M. Godin, O. Laroche, V. Tabard-Cossa, and P. Grütter, "Calibrating laser beam deflection systems for use in atomic force microscopes and cantilever sensors," Appl. Phys. Lett., vol. 88, 83108, 2006, http://dx.doi.org/10.1063/1.2177542

[22] A. Putman, B. de Grooth, N. van Hulst and J. Greve, "A detailed analysis of the optical beam deflection technique for use in atomic force microscopy," J. Appl. Phys., vol. 72, no. 1, pp. 6-12, 1992, http://dx.doi.org/ 10.1063/1.352149

[23] A. P. Gregory, J. F. Blackburn, K. Lees, R. N. Clarke, T. E. Hodgetts, S. M. Hanham and N. Klein, "A nearfield scanning microwave microscope for measurement of the permittivity and loss of high-loss materials", $84^{\text {th }}$ Microwave Measurement Conference (ARFTG), 2014, http://dx.doi.org/10.1109/ARFTG.2014.7013419

[24] B. Techaumnat and T. Takuma, "Analysis of the electric field and force in an arrangement of a conducting sphere and a plane electrode with a dielectric barrier," IEEE Trans. Diel. Electr. Insul., vol 13, no. 1, pp. 336344, 2006, http://dx.doi.org/ 10.1109/TDEI.2006.1624278

[25] D. Kimber, R. Pullar and N. Alford, "The effects of dielectric loss and tip resistance on resonator Q of the scanning evanescent microwave microscopy (SEMM) probe,” Meas. Sci. Tech., vol. 19, pp. 115502, 2008, http://dx.doi.org/10.1088/0957-0233/19/11/115502

[26] L. Chen, C. Ong, C. Neo, V. Varadan and V. Varadan, "Microwave Electronics: Measurement and Materials Characterization", Wiley 1994.

[27] R. E. Collin, "Field theory of guided waves", $2^{\text {nd }}$ Ed. Wiley 1991. pp. 388-9.

[28] W. B. Westphal, Dielectric constant and loss data, MIT report AFML-TR-250 Pt IV, 1980.

[29] J. Krupka, A. P. Gregory, O. C. Rochard, R. N. Clarke, B. Riddle and J. Baker-Jarvis, "Uncertainty of complex permittivity measure-ments by split-post dielectric resonator technique", J. Eur. Cer. Soc., vol. 21, no. 15, pp. 2673-2676, 2001, http://dx.doi.org/10.1016/S0955-2219(01)00343-0

[30] E. Hollmann, O. Vendik, A. Zaitsev and B. Melekh, "Substrates for high-Tc superconductor microwave integrated circuits," Supercond. Sci. and Technol., vol. 7, pp. 609-622, 1994, http://dx.doi.org/10.1088/09532048/7/9/001

[31] "The expression of uncertainty and confidence in measurement", Technical Report M3003, United Kingdom Accreditation Service (Feltham, UK). 